\title{
Associative and nonassociative schema theories of learning
}

\author{
ASGHAR IRAN-NEJAD \\ The University of Alabama, Tuscaloosa, Alabama
}

\begin{abstract}
Association, as the most basic unit of thinking and learning, has been a widely accepted, albeit controversial, concept in psychology. Recently, associationism has gained considerable momentum in the brain-inspired connectionist approach to learning. This paper compares connectionism with a nonassociative brain-inspired approach and reviews data bearing on the comparison regarding the nature of incompatibility between schemas.
\end{abstract}

In his book, Remembering, Bartlett (1932) argued against associationism and for a nonassociative cognitive approach. Bartlett's schema theory has had a major impact on current cognitive thinking. However, modern cognitive psychology, from its inception in the 1960s to today (Neisser, 1967; Rumelhart, Smolensky, McClelland, \& Hinton, 1986), has always been closer to pre-Bartlett associationism than to Bartlett's nonassociative theory (Iran-Nejad, 1980, 1987). Although associative and nonassociative views have generated much controversy (Jenkins, 1974), they have seldom been compared empirically. The purpose of the research reviewed here was to fill this gap. The associative view discussed here is the connectionist parallel distributed processing (PDP) schema theory of Rumelhart and colleagues (Rumelhart et al., 1986), and the nonassociative view is a Bartlettian biofunctional schema theory (Iran-Nejad \& Ortony, 1984).

\section{HISTORICAL NOTE}

It has been noted that many modern schema theories are abstractive-trace theories (diSibio, 1982). Ironically, a paper that Rumelhart (1980), the most outspoken architect of modern schema theory, wrote to clarify the schema notion was his last paper on conventional schema theory. Soon after the publication of this often-cited paper, which defined schemas as monolithic building blocks of cognition, Rumelhart abandoned his influential schema theory and turned to associative network modeling.

The first product of this shift in perspective was the word perception model (McClelland \& Rumelhart, 1981), which continued to view mental patterns as monolithic templates. Soon, however, Rumelhart disavowed the monolithic view to turn to parallel distributed processing (McClelland \& Rumelhart, 1985). He finally returned to the concept of schema and presented a connectionist analysis of it in the PDP tradition:

I wish to thank Genie Davis for assistance in the preparation of the manuscript. This research was supported in part by a University of Alabama (RGC PN 1437) and a College of Education research grant. Send reprint requests to Asghar Iran-Nejad, University of Alabama, Behavioral Studies, Box 870231, Tuscaloosa, AL 35487.
It was struggling with the concept of the schema and some of its difficulties that led one of us (DER) to an exploration of PDP models to begin with ... [and] it was therefore with some priority that we began to develop an interpretation of the schema in the language of parallel distributed processing. (Rumelhart et al., 1986, pp. 7-8)

Clearly, schema theories that defined the schema as a monolithic template were facing severe problems; however, not everyone viewed the schema in this way. In fact, I was able to show (Iran-Nejad, 1980) that Bartlett's theory did not have the problems associated with Rumelhart's early schema theory and that it had many of the features later found attractive in the PDP approach. For example, Rumelhart et al. (1986) noted, "One important difference between our interpretation of schemata and the more conventional ones is that in the conventional story, schemata are stored in memory. ... In our case, nothing stored corresponds very closely to a schema", (p. 21). However, as its title shows, this very point was the central theme of the Iran-Nejad (1980) paper.

Here, I concentrate on one fundamental difference between the Bartlettian biofunctional schema theory and the reinterpreted PDP schema theory: The former is nonassociative, whereas the latter is associative. In PDP schema theory, instead of monolithic schemas, "what is stored is a set of connection strengths which, when activated, have implicitly in them the ability to generate states that correspond to instantiated schemata" (Rumelhart et al., 1986, p. 21). In biofunctional schema theory, nothing at all is stored in the form of connections; schemas are ongoing patterns created and upheld from moment to moment by simultaneous activity in distributed constellations of brain microsystems.

Iran-Nejad (1980) used the term constellation to emphasize the nonassociative nature of brain functioning, and the light-bulb analogy to show how nonassociative structures can emerge out of simultaneous activity in distributed constellations of functionally autonomous microsystems. In a uniformly spread pattern of light, resulting from the simultaneous burning of a constellation of color-coded light bulbs, there are no discrete connection lines, and creation is a more meaningful way than storage to charac- 
Table 1

Basic Assumptions of Associative PDP and Nonassociative Biofunctional Schema Theories about Long-Term Remembering (1,2), the Nature of Mental Schemas and Knowledge $(3,4)$, the Role of Physiology $(5,6)$, the Nature of Self-Regulation and Learning Processes $(7,8)$, Their Approach to the Study of Cognition $(9,10)$, and Their Goals (11)

\section{Associative Schema Theory}

1. Long-term memory (LTM) traces represent static, feature-like, conceptual units and connections.

2. Remembering is a patchwork reinstatement of prior patterns of activation.

3. Mental schemas are activated patterns of prestored connection weights.

4. Knowledge is connection.

5. Mental units form local modules and talk directly only to immediate neighbors.

6. The neural network is a storehouse for superposed layers of connection weights and a medium for spread of activation in connection weight patterns.

7. Two sources of control regulate activity of the system: external or data-driven and internal or connection-driven.

8. Learning is the passive storage of static connection weight increments.

9. Knowledge is directly formalizable.

10. Mind is complex; isolate to simplify.

11. Discovering input-output transformations and organization of superposed layers of connection weights are the main goals.
Nonassociative Schema Theory

1. No static conceptual traces of any kind are stored; brain microsystems are the only LTM units that directly (re-)create and uphold the mind.

2. Remembering is fresh re-creation of prior mental states in new contexts; the past seldom reduplicates itself.

3. Mental schemas are ongoing awareness patterns, directly created, re-created, and upheld by brain microsystems.

4. Knowledge is ongoing awareness.

5. Brain microsystems are functionally autonomous and can talk to one another regardless of their physical location.

6. The neural network is a general-purpose communication medium in the same way the blood circulation system is a general-purpose medium for transporting oxygen and other substances, not to specific sites, but to every part of the body.

7. Three sources of control regulate the functioning of the system: external or stimulation-based, active or effort-based, and dynamic or interest-based (see Table 1).

8. The nervous system is a multiple-source system and learns when various learning and control processes operate simultaneously.

9. Knowledge is directly unanalyzable.

10. Simplification by isolation is the wrong way to simplify.

11. Discovering system functional properties responsible for the creation and upholding of ongoing schemas and the interaction between control and learning processes are the main goals. terize the relationship between the light bulb and the light it generates. The biofunctional model assumes that the brain creates the mind in an analogous fashion. Note that functional independence allows individual microsystems to participate in an indefinite number of schemas. It also means that for "two patterns that share elements, when one is functional the other is non-existent and vice versa" (Iran-Nejad, 1980, p. 14).

\section{BIOFUNCTIONAL SCHEMA THEORY}

PDP and biofunctional schema theories are compared in Table 1. As the left column shows, the assumption that knowledge is in the connections rather than in the units themselves is the foundation of the PDP model. Moreover, the principal goal of the model is to specify the process by which knowledge is stored, as connection weight increments, in readily retrievable formats. By contrast, connection strength is not a component of the model shown on the right side, and brain microsystems create and recreate knowledge rather than store it. Comprehension, learning, and remembering occur as a result of the simultaneous operation of attention, inquiry, closure, combination, and information-creation processes under the control of external (stimulation-based), active (effort-based), and dynamic (interest-based) sources of self-regulation.

Understanding how functional properties of the nervous system make this possible is the goal of nonassociative schema theory. The basic idea is that the brain is popu- lated by a vast number of specialized microsystems. These microsystems cannot be equated with knowledge units any more than a light bulb-as a physical system-can be equated with the light it creates. However, they can be called, metaphorically, knowledge microsystems since their function is to create knowledge much in the same sense that light bulbs can be called light bulbs, or bulbs whose function is to create light.

The light-bulb analogy suggests an intriguing way of conceptualizing how the brain creates knowledge. In a population of color-coded light bulbs, two very different types of activity can occur: (1) light bulbs can stay on, or (2) if so designed, they can produce sudden blinks. By analogy, the core assumption in the biofunctional model is that the brain can create two primary types of knowledge. It can create what might be called thematic knowledge by having large constellations of its microsystems stay on and engage in simultaneous ongoing activity, or it can create categorical or unit-level knowledge by having relatively smaller constellations of its microsystems produce, like the units in the traffic arrow on the freeway, sudden blinks. The details of how the brain can create these types of knowledge or use them to create propositional or sequence-level knowledge without storing static traces have been discussed in IranNejad and Ortony (1984). The main idea is that construction is possible because the brain is a multisource system. We remember, for example, not because the brain contains a unitary retrieval mechanism, but because it 
Table 2

Active and Dynamic Self-Regulation of Learning Processes

\begin{tabular}{llll}
\hline & \multicolumn{3}{c}{ Sources of Control } \\
\cline { 2 - 4 } Learning Processes & External & Active & Dynamic \\
\hline Attention & & & \\
1. Attention Catching & High & Low & Low \\
2. Attention Paying & Low & High & Low \\
3. Attention Holding & Low & Low & High \\
Inquiry & & & \\
1. Surprise & High & Low & Low \\
2. Self-Questioning & Low & High & Low \\
3. Curiosity & Low & Low & High \\
Closure & & & \\
1. Orientation & High & Low & Low \\
2. Prediction & Low & High & Low \\
3. Postdiction & Low & Low & High \\
Combination & & & \\
1. Independent & High & Low & Low \\
2. Sequential & Low & High & Low \\
3. Simultaneous & Low & Low & High \\
Information Creation & & & \\
1. Categorical & High & Low & Low \\
2. Propositional & Low & High & Low \\
3. Thematic & Low & Low & High \\
\hline
\end{tabular}

draws upon many sources that contribute to remembering as a re-creative process (Table 2).

Briefly, external, active, and dynamic sources of selfregulation interact with attention, inquiry, closure, combination, and information-creation processes to guide comprehension, learning, and remembering. External factors influence (via stimulation) attention catching, surprise, orientation, subconstellation independent functioning (blinking), and categorical knowledge-creation processes (Group 1); active internal factors regulate (via effort) attention paying, self-questioning, prediction, sequential functioning, and propositional knowledgecreation processes (Group 2); and dynamic internal factors regulate (via interest) attention holding, curiosity, postdiction, ongoing simultaneous functioning, and thematic knowledge-creation processes (Group 3). In a recent study, Iran-Nejad and Chissom (1988) obtained evidence suggesting that only Group 3 factors contribute directly to learning.

\section{Nonassociative Schema Construction}

How do self-regulation sources and learning processes interact to construct and uphold ongoing mental schemas? Although schema construction is a continuous process, and although all control sources and learning processes operate simultaneously all the time, it is possible to draw a global picture of the major landmarks involved. These landmarks are shown in Table 3 and are illustrated using story comprehension. Early in the story, comprehension is little more than generation of unit-level categorical knowledge. External and active (e.g., effort after meaning) sources of control engage Group 1 and Group 2 learning processes, which regulate word or sentence perception. The basic learning process at this stage is indepen- dent functioning (or blinking) of microsystem constellations. As constellations blink, one after another, they (1) generate categorical knowledge; (2) announce (broadcast) their themes, which circulate, instantaneously, in the nervous system; and (3) activate more theme-related microsystems. Not all announced themes, however, reach completion. Theme completion requires simultaneous functioning of a large number of theme-related microsystems to uphold an ongoing theme dynamically (e.g., without executive monitoring). After theme completion, which represents a major shift from external to dynamic sources of control and from Groups 1 and 2 to Group 3 processes, story comprehension involves such integration processes as curiosity, suspense, interest, need for schema closure, and uncertainty about story details. Further independent functioning, now occurring in the context of an ongoing theme, provides the details and directs comprehension toward categorical completion and final closure.

\section{Schema Similarity and Incompatibility}

Associative theories define similarity at the level of schema elements. Schemas are similar to the extent that they share elements, and different to the degree that they do not. Moreover, two schemas are incompatible to the degree that their elements are (1) different and (2) linked by mutual inhibition connections (Rumelhart et al., 1986, pp. 8-13). The biofunctional model implies that similarity cannot be defined at the level of elements alone because, as Table 4 shows, sharing elements is neither necessary nor sufficient. Two schemas are related by similarity to the degree that they share elements and have the same theme, by metaphoricity to the extent that they do not share elements while having the same theme, by incompatibility to the extent that they share elements while having different themes, or by difference to the extent that they do not share elements and have different themes.

Thus, associative theory implies that schemas that share elements are similar. Nonassociative theory implies that they can be either similar or incompatible depending on the thematic knowledge they contain. Associative theory implies that two incompatible schemas coexist, one in a state of activation and one in a state of inhibition; the mutual inhibition connection makes them incompatible. The nonassociative theory says that incompatible schemas cannot coexist because they are automatically mutually exclusive: When the same set of microsystems must participate in the upholding of two thematically different

Table 3

Schema Creation Landmarks in Story Comprehension

\begin{tabular}{lll}
\multicolumn{1}{c}{ Landmark } & $\begin{array}{c}\text { Primary } \\
\text { Processes }\end{array}$ & \multicolumn{1}{c}{ Example } \\
\hline Category Generation & Group 1 & Word Perception \\
& Group 2 & Sentence Perception \\
Theme Announcement & Group 1 & Topic Generation \\
Theme Completion & Group 3 & Schema Formation \\
Theme Integration & Group 3 & Schema Instantiation \\
Categorical Completion & Group 3 & Closure at Ending \\
\hline
\end{tabular}


Table 4

Elemental and Thematic Determinants of Between-Schema Relations

\begin{tabular}{lll}
\hline & \multicolumn{3}{c}{ of Between-Schema Relations } \\
\cline { 2 - 3 } Elements & Same & Different \\
\hline Shared & Similar & Incompatible \\
Not Shared & Metaphor & Different \\
\hline
\end{tabular}

schemas, at the time the microsystems are busy upholding one, the other cannot exist.

\section{EMPIRICAL COMPARISON OF THE TWO HYPOTHESES}

Mutual inhibition (MI) and exclusion (ME) hypotheses were first compared empirically by Diener and Iran-Nejad (1986). It was hypothesized that positive and negative affects were incompatible experiences, not because of separate underlying affective systems connected by mutual inhibition, but because the same brain components had to participate in upholding both affects, in the same fashion that a frown and a smile are incompatible behaviors to the extent that the same set of muscles must participate in both. Findings supported the ME hypothesis.

Further support for ME was obtained in another study (Iran-Nejad, 1988) using surprise-ending stories. In one experiment, evidence was obtained indicating that a surprise-ending story can create and uphold, in the manner discussed earlier, two mutually incompatible schemas, one prior to and one after the surprise. Two more experiments produced data supporting the hypothesis that the two incompatible schemas were created, one right after another, by the same set of idea units.

In the first experiment, subjects who read the surpriseending story rated sentences such as "Gabriel threatened Marilyn with a gun" as inconsistent with the overall story, and sentences such as "Gabriel protected Marilyn with a gun" as consistent with it. The opposite pattern of results was obtained from a control group that read the same story without the surprising ending. In a subsequent experiment, different groups of subjects read and recalled the above no-surprise and surprise-ending stories. As predicted by the ME hypothesis, there was a high positive correlation between recall data across the two incompatible schemas. The mutual inhibition hypothesis would have predicted a negative correlation.

\section{SUMMARY AND CONCLUSIONS}

The distinction between associative and nonassociative views has always been a metatheoretical controversy. This paper operationalized the difference theoretically and empirically. The basic idea is that instead of storing connection strength information, brain microsystems engage in two qualitatively different types of activity to create two qualitatively different types of knowledge. The nonassociative view gives a comprehensive account of how control and learning processes interact to build ongoing schemas and how different schemas relate by similarity and incompatibility.

\section{REFERENCES}

Bartlett, F. C. (1932). Remembering. Cambridge, England: Cambridge University Press.

DIENER, E., \& IRAN-NEJAD, A. (1986). The relationship in experience between various types of affect. Journal of Personality \& Social Psychology, 50, 1031-1038.

DISiBio, M. (1982). Memory for connected discourse: A constructivist view. Review of Educational Research, 52, 149-174.

IRAN-NEJAD, A. (1980, February). The schema: A structural or a functional pattern (Tech. Rep. No. 159). Urbana: University of Illinois, Center for the Study of Reading.

IRAN-NEJAD, A. (1987). The schema: A long-term memory structure or a transient functional pattern. In R. J. Tierney, P. Anders, \& N. Mitchell (Eds.), Understanding readers' understanding. Hillsdale, NJ: Erlbaum.

IRAN-NEJAD, A. (1988). Simultaneous and sequential aspects of schema construction during the comprehension of surprise-ending stories. Manuscript submitted for publication.

Iran-Nejad, A., \& Chissom, B. (1988). Active and dynamic selfregulation learning processes. Manuscript submitted for publication.

Iran-NeJAD, A., \& OrTony, A. (1984). A biofunctional model of distributed mental content, mental structures, awareness, and attention. The Journal of Mind \& Behavior, 5, 171-210.

Jenkins, J. J. (1974). Remember that old theory of memory? Well, forget it. American Psychologist, 29, 785-795.

McClelland, J. L., \& Rumelhart, D. E. (1981). An interactive activation model of context effects in letter perception: Part 1. An account of basic findings. The Psychological Review, 88, 375-407.

McClelland, J. L., \& Rumelhart, D. E. (1985). Distributed memory and the representation of general and specific information. Journal of Experimental Psychology: General, 114, 159-188.

Neisser, U. (1967). Cognitive psychology. New York: AppletonCentury-Crofts.

RumelharT, D. E. (1980). Schemata: The building blocks of cognition. In R. J. Spiro, B. C. Bruce, \& W. F. Brewer (Eds.), Theoretical issues in reading comprehension (pp. 33-58). Hillsdale, $\mathrm{NJ}$ : Erlbaum.

Rumelhart, D. E., Smolensky, P., McClelland, J. L., \& Hinton, G. E. (1986). Schemata and sequential thought processes in PDP models. In J. L. McClelland, D. E. Rumelhart, \& the PDP Research Group (Eds.), Parallel distributed processing (Vol. 2, pp. 7-57). Cambridge, MA: MIT Press. 